\title{
Chondrocutaneous transposition flap for congenital tragal malformation with dystopic cartilage
}

\author{
Ji-In Jeon, \\ Jeong Hyun Ha, \\ Sukwha Kim \\ Department of Plastic and \\ Reconstructive Surgery, Seoul National \\ University Hospital, Seoul National \\ University College of Medicine, Seoul, \\ Korea
}

Due to the variety in the shape of dysmorphic cartilage, tragus reconstruction is one of the most challenging goals in otoplasty. The authors describe a method to reconstruct a prominent tragus in a simple way suitable for accounting for the size, shape, and location of the remaining ear. We present a case of tragus deformity in an 11-year-old female patient after a previous excision of pretragal skin tags. There was a small remnant of the deeply located dystopic cartilage in a horizontal orientation. The dystopic cartilage was used to reconstruct the tragus using a chondrocutaneous transposition flap. Only a small portion of the pre-existing cartilage was used to create a chondrocutaneous transposition flap that supplemented the portion of cartilage during tragus reconstruction. The result was a new tragus that showed acceptable improvement in shape, location, and projection. Patients with a small portion of pre-existing cartilage near the tragal wall may benefit from the use of this method for tragus reconstruction.

Keywords: Abnormalities / External ear / Surgical flap

\section{INTRODUCTION}

Various abnormalities of the tragus can result from maldevelopment of the first and second branchial arches. The external portion of the ear arises from the first two branchial arches, which are located near the first branchial groove. Three hillocks form from the first branchial (or mandibular) arch and another three hillocks form the second branchial (or hyoid) arch. The first hillock has two portions: a ventral portion and a dorsal portion. An attempt has been made to correlate the presented malformation with the embryologic-fetal development of tragus. It was reported that hillock 1 forms the tragus, hillocks 2 and 3 form the helix, hillocks 4 and 5 form the antihelix and scapha, and hillock 6 forms the lobule [1]. Streeter [2] reported that the tragus and anterior crus of the helix are derived from

\footnotetext{
Correspondence: Sukwha Kim

Department of Plastic and Reconstructive Surgery, Seoul National University

Hospital, Seoul National University College of Medicine, 101 Daehak-ro, Jongno-gu,

Seoul 03080, Korea

E-mail: kimsw@snu.ac.kr

Received October 4, 2019 / Revised October 28, 2019 / Accepted December 30, 2019
}

the mandibular arch, while Wood-Jones and I-Chuan [3] reported that only the tragus derives from the mandibular arch. The most common forms of tragal malformation are skin tags, accessory tragus, macrotragus, preauricular sinus, and cysts.

The degree of congenital malformations of the tragus leads to variations in size, shape, and location, and it is important for diagnosis and treatment. It is difficult to choose an appropriate method that can result in an ideal shape. The goals of tragus reconstruction in congenital tragal malformation are to create a good tragus projection, hide the external meatus, and leave an inconspicuous scar [4]. We developed a technique to create a prominent tragus using dystopic cartilage near the tragal wall in a simple way. The result of this technique has proved acceptable and the procedures are described in detail below.

\section{CASE REPORT}

An 11-year-old female patient presented with congenital tragal malformation after a previous excision of pretragal skin tags. She only had a small portion of horizontally lying cartilage near 
the tragal area after the previous excision of the skin tags. Superior to this accessory cartilage, there was a triangular-shaped depression with band-like structure from the helical root to the medial end of the dystopic cartilage. She desired an improved appearance. Her preoperative photo is shown in Fig. 1.

A single-stage reconstructive procedure with a transposition technique using a chondrocutaneous flap was performed. A Cshaped incision (Fig. 2, a) around the horizontally lying pre-existing cartilage was made and dissection was performed towards the proposed tragus and a posteriorly based pre-existing cartilage flap was created. The subcutaneous soft tissue and remaining cartilaginous portion were preserved to reconstruct the tragus. This composite flap was transposed $90^{\circ}$ upwards using the posterior part of the flap as a pedicle.
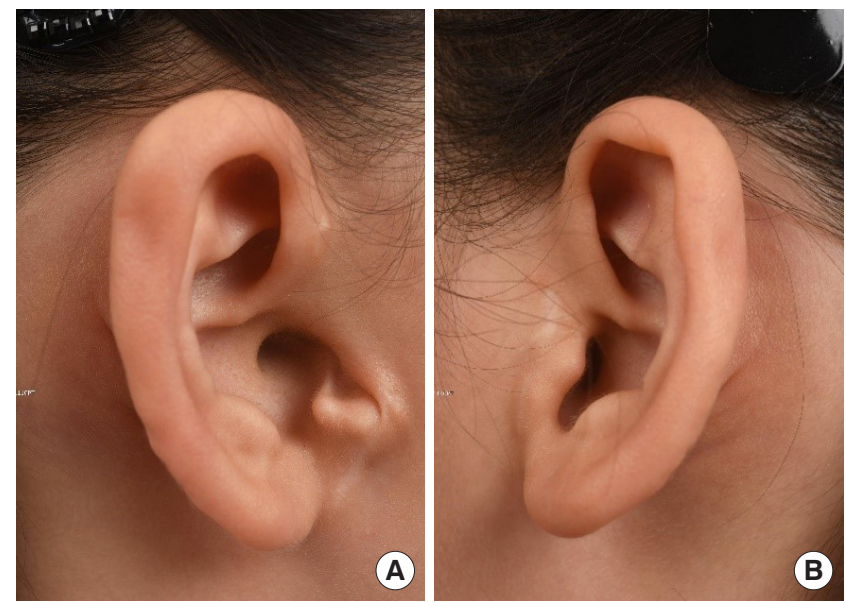

Fig. 1. An 11-year-old girl presented a tragal malformation of right ear, showing a deformed small horizontally lying cartilage. (A) Right ear. (B) Left ear.

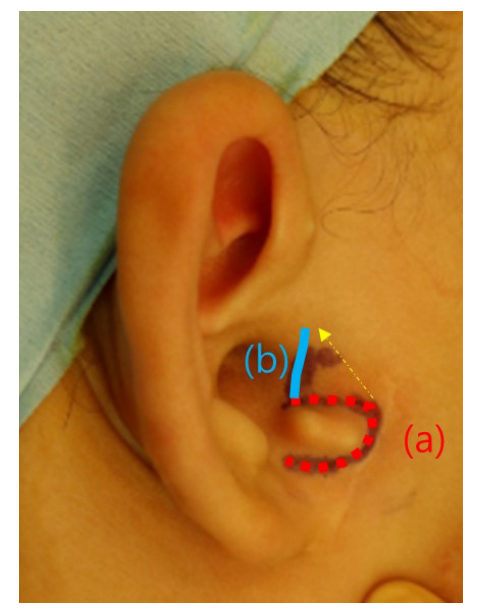

Fig. 2. Intraoperative view. A single-stage reconstructive procedure with transposition technique using a chondrocutaneous flap: (a) Cshaped incision around horizontally lying dystopic cartilage and (b) vertical linear incision along tragal wall.
A vertical linear incision (Fig. 2, b) along the tragal wall was made and the posteriorly based pre-existing cartilage flap was transposed to the desired vertical direction. The depressed area was then undermined to detach the skin from the underlying tethering. The chondrocutaneous flap was transpositioned under the depressed area and was then inserted at precisely the proposed location of the tragus and sutured to the anchoring points below the cartilage of the helical crus to avoid reversion to the previous direction. Trimming of the remnant skin flap after transposition was necessary to avoid soft tissue depression around the tragus. The remaining wound was closed with minimal tension. There should be no tension in the repaired site in order to prevent the reconstructed tragus from becoming distorted. A prominent tragus with a gentle convex border was created using this procedure (Fig. 3).

The patient returned for follow-up and was content with the improvement at 2 months postoperatively (Fig. 4).

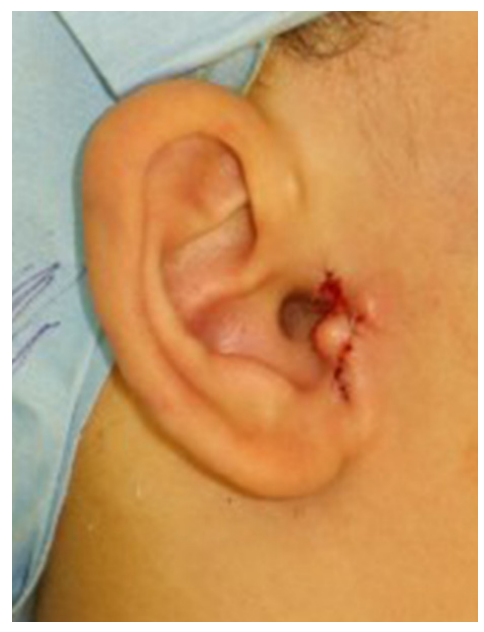

Fig. 3. Immediate postoperative view.

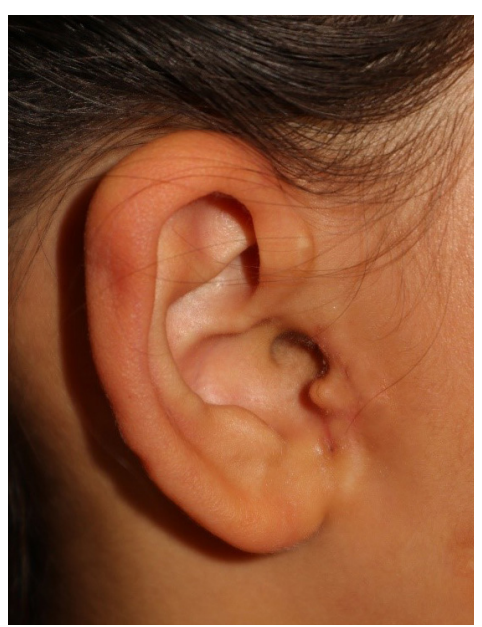

Fig. 4. Two-month postoperative view. 


\section{DISCUSSION}

We encountered tragal deformity with tragal malformation and achieved an acceptable result using a small portion of horizontally located dystopic cartilage near the tragal wall. Although the remnant tissue was too little to reconstruct the tragus, reconstruction could be completed in a single-stage with satisfactory improvement in shape and location. A single-stage reconstructive procedure with a transposition technique using a chondrocutaneous flap is an option that can reduce unnecessary procedures and donor site morbidity.

Various techniques to correct deformities of the contour of tragus have previously been described [5]. More complicated or multistage methods using autogenous substances may also lead to excellent cosmetic results; however, they are not always necessary. Concha cartilage, rib cartilage, and dermofat are common autogenous materials used to correct tragal deformities. A disadvantage of using these materials is donor site morbidity. Tragal reconstruction using our method has the advantages of less donor site morbidity and shorter operation time.

The main limitation of this method is that it is difficult to obtain an optimal contour unless the volume of the residual cartilage is sufficient. Reconstruction using residual tissue is not possible in all cases [6]. To use this reconstruction method, it is important to select patients with mild deformities and sufficient remnant tissue. Patients with severe tragal deformity require treatment using other reconstruction techniques for satisfactory results. For satisfactory postoperative results, the patient's requirements should be understood and the patient should be provided with information about how the surgery will be performed and other options for reconstruction with their advantages and disadvantages before surgery.

In order to achieve an acceptable result using the remnant ear tissue, several details regarding the case need to be considered. A transposition technique using a chondrocutaneous flap can be used to account for a variety of sizes, shapes, and locations of a remnant ear to allow the reconstruction of a naturally shaped tragus with a good position. These methods can be safe and simple for tragal reconstruction in patients with an accessory tragus.

\section{NOTES}

\section{Conflict of interest}

No potential conflict of interest relevant to this article was reported.

\section{Ethical approval}

The study was approved by the Institutional Review Board of Seoul National University Hospital (IRB No. 1910-009-1067) and performed in accordance with the principles of the Declaration of Helsinki. Written informed consent was obtained.

\section{Patient consent}

The patient's parent provided written informed consent for the publication and the use of her images.

\section{ORCID}

Ji-In Jeon https://orcid.org/0000-0001-9596-386X

Jeong Hyun Ha https://orcid.org/0000-0002-0927-4185

Sukwha Kim https://orcid.org/0000-0002-9291-2634

\section{REFERENCES}

1. His W. Die formentwicklung des ausseren ohres: anatomie menschlicher embryonen, part III. Leipzig: F.C.W. Vogel; 1885.

2. Streeter GL. Development of the auricle in the human embryo. Contrib Embryol 1992;14:111-38.

3. Wood-Jones F, I-Chuan W. The development of the external ear. J Anat 1934;68(Pt 4):525-33.

4. Porter CJ, Tan ST. Congenital auricular anomalies: topographic anatomy, mbryology, classification, and treatment strategies. Plast Reconstr Surg 2005;115:1701-12.

5. Gore SM, Myers SR, Gault D. Mirror ear: a reconstructive technique for substantial tragal anomalies or polyotia. J Plast Reconstr Aesthet Surg 2006;59:499-504.

6. Perez CF, Gaball CW. Functional and aesthetic tragal reconstruction in the age of mobile electronic devices. Case Rep Otolaryngol 2016;2016:2591705. 